\title{
Complete Response in cGVHD
}

National Cancer Institute

\section{Source}

National Cancer Institute. Complete Response in CGVHD. NCI Thesaurus. Code C103139.

Complete resolution of all signs and symptoms in all affected org ans or tissues in chronic Graft versus Host Disease (cGVHD). 